International Journal of Pure and Applied Mathematics

Volume 93 No. 5 2014, 625-628

ISSN: 1311-8080 (printed version); ISSN: 1314-3395 (on-line version)

url: http://www.ijpam.eu

doi: http://dx.doi.org/10.12732/ijpam.v93i5.3

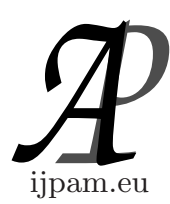

\title{
AN IMPROVED NEGATIVE BINOMIAL TO APPROXIMATE THE PÓLYA DISTRIBUTION
}

\author{
K. Teerapabolarn \\ Department of Mathematics \\ Faculty of Science \\ Burapha University \\ Chonburi, 20131, THAILAND
}

\begin{abstract}
This paper gives an improved negative binomial distribution with parameters $r$ and $p$ to approximate the Pólya distribution with parameters $N, m$ and $r$, where $p=1-q=\frac{N}{N+m}$. The improved approximation is more accurate than the negative binomial approximation when $N$ is sufficiently large.
\end{abstract}

AMS Subject Classification: 62E17, 60F05

Key Words: negative binomial approximation, negative binomial probability function, Pólya probability function

\section{Introduction}

A ball is drawn at random from an urn containing $r$ blue balls and $N-r$ red balls. If a blue ball is drawn, then replace it together with another blue ball into this urn, and similarly for a red ball. Do this way for $m$ draws and let $X$ be the number of blue balls drawn in the $m$ drawings, then the distribution of $X$ is a Pólya distribution with parameters $N, m$ and $r$, and its probability function is given by

$$
\operatorname{py}_{N, m, r}(x)=\frac{\left(\begin{array}{c}
r+x-1 \\
x
\end{array}\right)\left(\begin{array}{c}
N-r+m-x-1 \\
m-x
\end{array}\right)}{\left(\begin{array}{c}
N+m-1 \\
m
\end{array}\right)}, x=0,1, \ldots, m .
$$

The mean and variance of $X$ are $E(X)=\frac{r m}{N}$ and $\operatorname{Var}(X)=\frac{r m(N+m)(N-r)}{N^{2}(N+1)}$,

Received: December 25, 2013

(C) 2014 Academic Publications, Ltd. url: www.acadpubl.eu 
respectively. If $N \rightarrow \infty$ while $\frac{m}{N}$ remains a constant, then $\mathbf{p y}_{N, m, r}(x) \rightarrow$ $\mathbf{n b}_{r, p}(x)=\left(\begin{array}{c}r+x-1 \\ x\end{array}\right) p^{r} q^{x}$ for every $x \in\{0, \ldots, m\}$, where $p=1-q=\frac{N}{N+m}[2]$. Therefore, the negative binomial probability function can be used as an estimate of the Pólya probability function when $N$ is large. In this case, Malingam and Teerapabolarn [2] gave a bound on $\left|\mathbf{p y}_{N, m, r}(x)-\mathbf{n b}_{r, p}(x)\right|$ for $x \in\{0, \ldots, m\}$.

In this paper, we focus on giving an improved negative binomial probability function, $\widehat{\mathbf{n b}}_{r, p}(x)$, to approximate the Pólya probability function, and the accuracy of the approximation is measured in the form of $\left|\mathbf{p y}_{N, m, r}(x)-\widehat{\mathbf{n b}}_{r, p}(x)\right|$ for $x \in\{0, \ldots, m\}$. The result of this study is in Section 2. In Section 3, some numerical examples are given to illustrate the improved approximation and the conclusion of this study is presented in the last section.

\section{Result}

The following lemma is directly obtained from [1].

Lemma 2.1. For $x, N \in \mathbb{N}$ and $0<p<1$, then

$$
\begin{aligned}
& \prod_{i=1}^{x-1}\left(p-\frac{i}{N}\right)=p^{x}\left[1-\frac{x(x-1)}{2 N p}\right]+O\left(\frac{1}{N^{2}}\right), \\
& \frac{1}{\prod_{i=0}^{x-1}\left(1-\frac{i}{N}\right)}=1+\frac{x(x-1)}{2 N}+O\left(\frac{1}{N^{2}}\right) .
\end{aligned}
$$

Theorem 2.1. Let $x \in\{0, \ldots, m\}, p=\frac{N}{N+m}$ and $r \leq \sqrt{2 N}-1$. Then we have the following:

$$
\mathbf{p y}_{N, m, r}(x)=\widehat{\mathbf{n b}}_{r, p}(x)+O\left(\frac{1}{(N+m)^{2}}\right)
$$

and for large $N$,

$$
\widehat{\mathbf{n b}}_{r, p}(x) \approx \mathbf{p y}_{N, m, r}(x),
$$

where $\widehat{\mathbf{n b}}_{r, p}(x)=\mathbf{n b}_{r, p}(x)\left\{1+\frac{(r+x+1)(r+x)}{2(N+m)}-\frac{(r+1) r}{2 N}\right\} /\left\{1+\frac{x(x-1)}{2 m}\right\}$.

$$
\text { Proof. Let } \eta=\left\{\begin{array}{ll}
0 & \text { if } x=0, \\
x-1 & \text { if } x=1, \ldots, m .
\end{array}\right. \text { Applying Lemma 2.1, it follows }
$$
that

$$
\mathbf{p y}_{N, m, r}(x)=\left(\begin{array}{c}
r+x-1 \\
x
\end{array}\right) \frac{[(N-1) \cdots(N-r)][m \cdots(m-\eta)]}{(N+m-1) \cdots(N+m-r-x)}
$$




$$
\begin{aligned}
= & \left(\begin{array}{c}
r+x-1 \\
x
\end{array}\right) \frac{\prod_{i=1}^{r}\left(p-\frac{i}{N+m}\right) \prod_{i=0}^{\eta}\left(q-\frac{i}{N+m}\right)}{\prod_{i=1}^{r+x}\left(1-\frac{i}{N+m}\right)} \\
= & \left(\begin{array}{c}
r+x-1 \\
x
\end{array}\right) \frac{p^{r} q^{x}}{1+\frac{x(x-1)}{2(N+m) q}}\left\{1+\frac{(r+x+1)(r+x)}{2(N+m)}\right. \\
& \left.-\frac{(r+1) r}{2(N+m) p}\right\}+O\left(\frac{1}{(N+m)^{2}}\right) \\
= & \frac{\mathbf{n b}_{r, p}(x)}{1+\frac{x(x-1)}{2 m}}\left\{1+\frac{(r+x+1)(r+x)}{2(N+m)}-\frac{(r+1) r}{2 N}\right\} \\
& +O\left(\frac{1}{(N+m)^{2}}\right) \\
= & \widehat{\mathbf{n b}}_{r, p}(x)+O\left(\frac{1}{(N+m)^{2}}\right) .
\end{aligned}
$$

Also, if $N$ is large, then $O\left(\frac{1}{(N+m)^{2}}\right) \approx 0$. Hence $\widehat{\mathbf{n b}}_{r, p}(x) \approx \mathbf{p y}_{N, m, r}(x)$.

\section{Numerical Examples}

The following examples are given to illustrate how well the improved negative binomial distribution approximates the Pólya distribution.

3.1. Let $N=50, m=10$ and $r=5$, then $p=\frac{50}{60}$ and the numerical results are as follows:

\begin{tabular}{cccccc}
\hline$x$ & $\mathbf{p y}_{N, m, r}(x)$ & $\widehat{\mathbf{n b}}_{r, p}(x)$ & $\mathbf{n b}_{r, p}(x)$ & $\left|\mathbf{p y}_{N, m, r}(x)-\widehat{\mathbf{n b}}_{r, p}(x)\right|$ & $\left|\mathbf{p y}_{N, m, r}(x)-\mathbf{n b}_{r, p}(x)\right|$ \\
\hline 0 & 0.38089033 & 0.38178369 & 0.40187757 & 0.00089337 & 0.02098725 \\
1 & 0.35267623 & 0.35164288 & 0.33489798 & 0.00103335 & 0.01777825 \\
2 & 0.17966525 & 0.17759741 & 0.16744899 & 0.00206784 & 0.01221626 \\
3 & 0.06449522 & 0.06511905 & 0.06511905 & 0.00062383 & 0.00062383 \\
4 & 0.01770457 & 0.01967138 & 0.02170635 & 0.00196681 & 0.00400178 \\
5 & 0.00382419 & 0.00526379 & 0.00651191 & 0.00143960 & 0.00268772 \\
6 & 0.00065037 & 0.00130238 & 0.00180886 & 0.00065201 & 0.00115849 \\
7 & 0.00008517 & 0.00030564 & 0.00047375 & 0.00022048 & 0.00038858 \\
8 & 0.00000815 & 0.00006909 & 0.00011844 & 0.00006093 & 0.00011028 \\
9 & 0.00000051 & 0.00001519 & 0.00002851 & 0.00001467 & 0.00002800 \\
10 & 0.00000002 & 0.00000327 & 0.00000665 & 0.00000325 & 0.00000664 \\
\hline
\end{tabular}

3.2. Let $N=100, m=30$ and $r=10$, then $p=\frac{100}{130}$ and the numerical results are as follows: 


\begin{tabular}{cccccc}
\hline$x$ & $\mathbf{p y}_{N, m, r}(x)$ & $\mathbf{n b}_{r, p}(x)$ & $\mathbf{n b}_{r, p}(x)$ & $\left|\mathbf{p y}_{N, m, r}(x)-\mathbf{n b}_{r, p}(x)\right|$ & $\left|\mathbf{p y}_{N, m, r}(x)-\mathbf{n b}_{r, p}(x)\right|$ \\
\hline 0 & 0.06335387 & 0.06333139 & 0.07253815 & 0.00002249 & 0.00918428 \\
1 & 0.15971565 & 0.16031360 & 0.16739573 & 0.00059796 & 0.00768008 \\
2 & 0.21588683 & 0.21589065 & 0.21246381 & 0.00000382 & 0.00342301 \\
3 & 0.20666089 & 0.20503501 & 0.19612044 & 0.00162589 & 0.01054045 \\
4 & 0.15633184 & 0.15416198 & 0.14709033 & 0.00216986 & 0.00924151 \\
5 & 0.09896485 & 0.09787600 & 0.09504298 & 0.00108886 & 0.00392187 \\
6 & 0.05425705 & 0.05469189 & 0.05483249 & 0.00043485 & 0.00057544 \\
7 & 0.02633970 & 0.02767935 & 0.02892263 & 0.00133965 & 0.00258293 \\
8 & 0.01149422 & 0.01295112 & 0.01418321 & 0.00145689 & 0.00268899 \\
9 & 0.00455627 & 0.00568778 & 0.00654610 & 0.00113151 & 0.00198983 \\
10 & 0.00165268 & 0.00237124 & 0.00287021 & 0.00071855 & 0.00121753 \\
11 & 0.00055135 & 0.00094654 & 0.00120428 & 0.00039518 & 0.00065293 \\
12 & 0.00016975 & 0.00036417 & 0.00048635 & 0.00019443 & 0.00031660 \\
13 & 0.00004832 & 0.00013575 & 0.00018993 & 0.00008743 & 0.00014161 \\
14 & 0.00001273 & 0.00004923 & 0.00007201 & 0.00003650 & 0.00005928 \\
15 & 0.00000310 & 0.00001743 & 0.00002659 & 0.00001433 & 0.00002348 \\
16 & 0.00000070 & 0.00000604 & 0.00000959 & 0.00000534 & 0.00000889 \\
17 & 0.00000015 & 0.00000205 & 0.00000338 & 0.00000191 & 0.00000324 \\
18 & 0.00000003 & 0.00000069 & 0.00000117 & 0.00000066 & 0.00000114 \\
\hline
\end{tabular}

From the examples 3.1 and 3.2, it can be seen that the improved negative binomial distribution is better than the negative binomial distribution.

\section{Conclusion}

In this study, an improved negative binomial distribution with parameters $r$ and $p=\frac{N}{N+m}$ was obtained. It is more appropriate for approximating the Pólya distribution, that is, the improved negative binomial distribution can be used as an estimate of the Pólya distribution when $N$ is sufficiently large. In addition, the improved approximation is more accurate than the negative binomial approximation.

\section{References}

[1] D.P. Hu, Y.Q. Cui, A.H. Yin , An improved negative binomial approximation for negative hypergeometric distribution, Applied Mechanics and Materials, 427-429 (2013), 2549-2553.

[2] P. Malingam, K. Teerapabolarn, A pointwise negative binomial approximation by $w$-functions, International Journal of Pure and Applied Mathematics, 69 (2011), 453-467. 